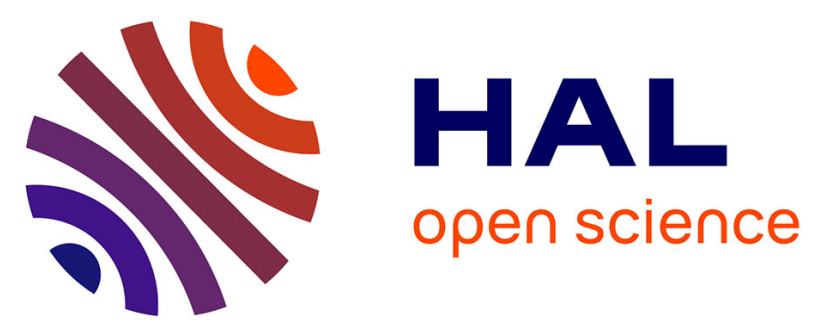

\title{
Spectral and temporal phase measurement by Optical Frequency-Domain Reflectometry
}

Bruno Robillart, Cosimo Calo, Abdoulaye Fall, François Lamare, Yaneck

Gottesman, Badr-Eddine Benkelfat

\section{- To cite this version:}

Bruno Robillart, Cosimo Calo, Abdoulaye Fall, François Lamare, Yaneck Gottesman, et al.. Spectral and temporal phase measurement by Optical Frequency-Domain Reflectometry. PHOTONICS WEST 2014: Fiber Lasers XI Conference, Feb 2014, San Francisco, CA, United States. pp.896134, 10.1117/12.2039600 . hal-01285646

\section{HAL Id: hal-01285646 \\ https://hal.science/hal-01285646}

Submitted on 9 Mar 2016

HAL is a multi-disciplinary open access archive for the deposit and dissemination of scientific research documents, whether they are published or not. The documents may come from teaching and research institutions in France or abroad, or from public or private research centers.
L'archive ouverte pluridisciplinaire HAL, est destinée au dépôt et à la diffusion de documents scientifiques de niveau recherche, publiés ou non, émanant des établissements d'enseignement et de recherche français ou étrangers, des laboratoires publics ou privés. 


\title{
Spectral and temporal phase measurement by Optical Frequency-Domain Reflectometry
}

\author{
Bruno Robillart ${ }^{a}$, Cosimo Calò ${ }^{b}$, Abdoulaye Fall $^{a}$, François Lamare ${ }^{a}$, Yaneck Gottesman ${ }^{a}$ and \\ Badr-Eddine Benkelfat ${ }^{a}$ \\ ${ }^{a}$ Institut Télécom, Télécom SudParis, SAMOVAR UMR INT-CNRS, 91011 Evry, France; \\ ${ }^{b}$ Laboratory for Photonics and Nanostructures LPN-CNRS, 91460 Marcoussis, France.
}

\begin{abstract}
The capability of measuring the spectral and temporal phase of an optical signal is of fundamental importance for the advanced characterization of photonic and optoelectronic components, biochemical sensors, structural monitoring sensors and distributed sensor networks. To address this problem, several techniques have been developed (frequency-resolved optical gating (FROG), spectral phase interferometry for direct electric-field reconstruction (SPIDER), stepped-heterodyne technique, laser Doppler vibrometry (LDV) and Doppler optical coherence tomography (OCT)). However, such techniques often lack of versatility for the mentioned applications. Swept-wavelength interferometric techniques and, among these, optical frequency-domain reflectometry (OFDR) are flexible and highly sensitive tools for complete characterization of amplitude and phase of target devices.

In this work, we investigate the spectral and temporal phase measurement capabilities of OFDR. Precise characterization of spectral phase information is demonstrated by retrieving the phase response of a commercial optical filter, the Finisar Waveshaper $1000 \mathrm{~S} / \mathrm{X}$, programmable in attenuation and phase over C+L band (1530$1625 \mathrm{~nm}$ ). The presented results show accurate retrieval of group delay dispersion (GDD) and discrete phase shift as well as filter attenuation profile. Although some intrinsic accuracy limitations of OFDR phase measurements may be encountered (and herein specified), we show that information encoded in OFDR reflectogram data is very rich when adequately exploited. In addition to previously published results, we demonstrate the high sensitivity of the technique to Doppler effects. From practical point of view, such sensitivity can be beneficially exploited for the characterisation of dynamical aspects of samples under test. Unlike LDV, OFDR allows the simultaneous retrieval of the temporal position of several localised reflecting target along the beam path. All these aspects make OFDR a highly promising candidate for the study of both static and dynamic aspects of complex photonic components or to probe a parallel sensor network, as needed for future applications.
\end{abstract}

Keywords: OFDR, Swept-wavelength interferometry, Spectral Phase, Group Delay Dispersion, Phase Modulation, Doppler effect

\section{INTRODUCTION}

The precise characterization of the amplitude and phase response of an optical component is of fundamental importance for the understanding of its properties and for the analysis of its performances. Phase features like discrete phase shifts or chromatic dispersion, in particular, may have a significant impact on the characteristics of optical devices and systems. Discrete optical phase shifts, for example, are typically used in the design of distributed feedback lasers to improve the single longitudinal mode property. ${ }^{1}$ On the other hand, group velocity dispersion (GVD) is a basic design parameter of fiber-optic transmission systems ${ }^{2}$ and significantly affects the four-wave mixing (FWM) efficiency in the formation of optical frequency combs in microresonators ${ }^{3}$ and the chirp and stability of mode-locked laser (MLL) pulses. ${ }^{4}$

In regard to the latter issue, several techniques have been developed in the context of research on ultra-short MLL pulses, advanced optical modulation formats and, recently, optical arbitrary waveform generation ${ }^{5}$ to fully characterize optical signals in amplitude and phase, such as frequency-resolved optical gating (FROG), ${ }^{6}$

Further author information: (Send correspondence to Yaneck Gottesman)

Yaneck Gottesman: E-mail: yaneck.gottesman@telecom-sudparis.eu, Telephone: +331 70220251 
frequency-resolved Mach-Zehnder gating (FRMZG), ${ }^{7}$ spectral phase interferometry for direct electric field reconstruction (SPIDER $)^{8}$ and stepped heterodyne technique. ${ }^{9}$ However, such techniques are typically used to measure the pulse shape and chirp of the output of a MLL, rather then the laser intracavity dispersion. Getting insight into the laser cavity properties is desirable for a deeper understanding of its dynamical aspects.

Interferometric techniques are well known to be very sensitive and versatile for the characterization of optical components and, for these reasons, they appear to be well suited to address the problem of measuring the amplitude and phase transfer function of optoelectronic devices. Indeed, techniques such as white light interferometry $^{10}$ and optical low-coherence reflectometry (OLCR) ${ }^{11}$ have already proven to be effective in determining the dispersion of both active ${ }^{12,13}$ and passive devices. ${ }^{11,14,15}$ However, these techniques often require a precise alignment of free-space optical components and use of mechanical translation stages to provide variable optical delays, with detrimental effects on the setup flexibility and the acquisition speed. Swept-wavelength interferometric techniques and, among these, coherent optical frequency-domain reflectometry (Co-OFDR) are flexible and highly sensitive tools for the complete characterization of optical components and have been widely used for the detection of discrete reflections and defects in optical fibers and integrated optical devices. ${ }^{16}$ Nonetheless, from a metrological point of view, some ambiguities have been identified ${ }^{16}$ in the past when exploiting the amplitude of recorded reflectograms.

In this paper, we aim at investigating the spectral phase measurement performances of coherent OFDR by evaluating the smallest dispersion values measurable by the tool. For this purpose, and because of the unavailability of group delay dispersion (GDD) or optical phase references, here we carry out experiments using a commercially available optical filter (Finisar Waveshaper $1000 \mathrm{~S} / \mathrm{X}$ ), for which the amplitude and phase transfer function can be precisely controlled in the $\mathrm{C}+\mathrm{L}$ band $(1530-1625 \mathrm{~nm})$. We demonstrate that discrete phase shifts down to $0.05 \mathrm{rad}$ and dispersion values down to $20 \mathrm{fs} / \mathrm{nm}$ (over $1 \mathrm{THz}$ optical frequency span) can be measured by Co-OFDR. In addition, we show that the smallest phase features measurable by OFDR is ultimately limited by parasitic phase fluctuations. Specific signatures of these fluctuation are identified and studied in detail. In particular, we report that environmental vibrations do affect OFDR measurements and introduce additional ambiguities to those previously reported in literature. ${ }^{16,17}$ Nonetheless, understanding of this phenomenon enables new uses of OFDR setup and new opportunities for characterization of dynamical objects.

The paper is organized as follows: in sec. 2 we introduce to the principles of OFDR technique and to the digital signal processing procedure necessary to extract the phase information; in sec. 3 we report on the spectral phase measurement by OFDR with the use of the programmable filter; in sec. 4 modeling and experimental evidence of phase modulation induced by environmental fluctuation are presented; finally, discussion and conclusions are drawn in sec. 5 .

\section{OFDR BASICS}

\subsection{OFDR Setup}

An implementation of an OFDR setup in transmission configuration (denoted in the following an OFDI setup for Optical Frequency Domain Interferometry) is sketched in fig. 1. The setup is essentially composed of two parts: a core part constituted by a measurement Mach-Zehnder interferometer, with the device under test (DUT) in one of its arms (in our case the programmable filter Finisar Waveshaper $1000 \mathrm{~S} / \mathrm{X}$ ) and an auxiliary reference Mach-Zehnder interferometer used for triggering data acquisition purposes. The light from a tunable laser source (TLS) constitutes the input of the system.

Assuming a time-linear frequency sweep $\nu(t)=\nu_{0}+\gamma t$ of the optical source, the electric field of the laser output can be written as:

$$
\mathbf{E}(t)=E_{0} \mathrm{e}^{\mathrm{j} \phi(t)}=E_{0} \mathrm{e}^{\mathrm{j} 2 \pi\left[\nu_{0}+(\gamma / 2) t\right] t} \boldsymbol{\varrho},
$$

where $E_{0}$ is a constant amplitude, $\varrho$ is a unit polarization vector, and $\gamma$ is the tuning rate of the instantaneous optical frequency $\nu(t)=(1 / 2 \pi)(\mathrm{d} \phi / \mathrm{d} t)$. After propagation through the measurement interferometer, the optical 


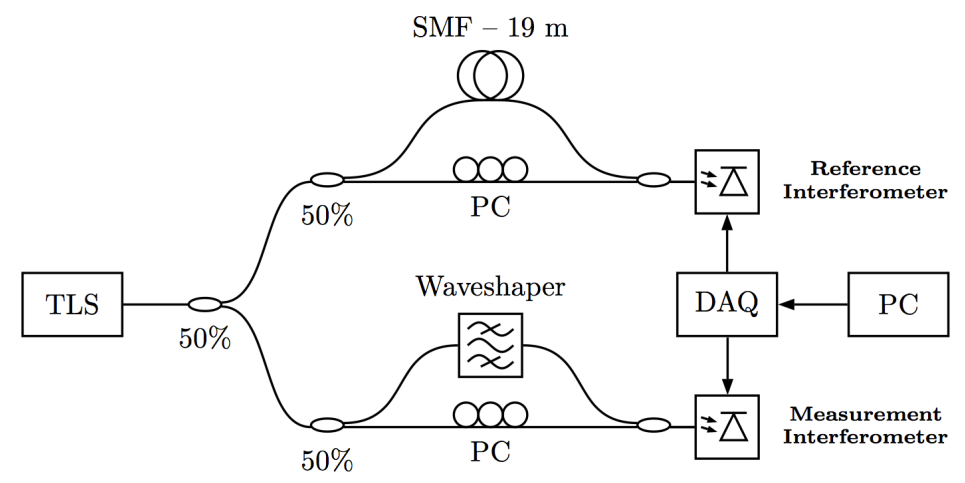

Figure 1. Setup for optical frequency-domain reflectometry with fiber optic components.

fields in the two paths of group delays $\tau_{1}$ and $\tau_{2}$, lead to a detector voltage given by:

$$
\begin{aligned}
U(t) & =U_{0}\left|\mathrm{e}^{\mathrm{j} \phi\left(t-\tau_{1}\right)}+H(\nu) \mathrm{e}^{\mathrm{j} \phi\left(t-\tau_{2}\right)}\right|^{2} \\
& =U_{0}\left[1+|H(\nu)|^{2}+2 \operatorname{Re}\left\{H(\nu) \mathrm{e}^{\mathrm{j}\left(2 \pi \gamma \tau_{0} t+\varphi\right)}\right\}\right] \\
& =U_{0}\left[1+A(\nu)^{2}+2 A(\nu) \cos \left(2 \pi \gamma \tau_{0} t+\Phi(\nu)+\varphi\right)\right],
\end{aligned}
$$

where $\tau_{0}=\tau_{2}-\tau_{1}, \varphi$ is a phase constant, $H(\nu)=A(\nu) \mathrm{e}^{\mathrm{j} \Phi(\nu)}$ is the transfer function of the DUT, $U_{0}=\sigma E_{0}^{2} / 2$ and $\sigma$ depends on the detector sensitivity. Here, for sake of simplicity, assumptions on the co-polarization of the optical fields from the two interferometer paths and on the polarization independence of the transfer function of the DUT are made. Although these assumptions are, in general, quite strong, they are reasonable for the particular case in exam. In fact, co-polarization of the interfering signals can be achieved over a wide wavelength range of several $\mathrm{nm}$ by adjusting the fringe visibility by means of the fiber polarization controller on one of the interferometer arms. If the two signals are not co-polarized, polarization fading of the interference fringes occurs; however polarization-diverse detection can be used to avoid it. ${ }^{16,18}$ On the other hand, the assumption on polarization independence of $H(\nu)$ is justified for the Waveshaper by the use of polarization-diverse optics. For further information on the filter, the interested reader is referred to Ref. 2.

Since in eq. 2, the DC term $U_{0}\left(1+A(\nu)^{2}\right)$ does not carry any useful information, it is typically neglected and fringe equation is more conveniently written as a function of the instantaneous optical frequency $\nu(t)$ as:

$$
\left.\tilde{U}(\nu)=2 U_{0} A(\nu) \cos \left(2 \pi \nu \tau_{0}+\Phi(\nu)+\xi\right)\right],
$$

with $\xi=\varphi-2 \pi \nu_{0} \tau_{0}$. In other words, the interference fringes recorded at the output of the measurement interferometer are amplitude modulated by the amplitude transfer function of the DUT and phase modulated by its phase transfer function. By sampling fringes at constant frequency spacing $\delta \nu$ and applying fast Fourier transform (FFT) to the sampled signal, one can obtain a positive frequency peak centered at the time delay $\tau_{0}$. Such peak contains all the information on the DUT. A negative frequency peak at $-\tau_{0}$ will also be present because of the hermitian properties of the FFT applied on a real signal. In case light travels along multiple paths with different lengths in the measurement arm of the interferometer, different peaks will be present in the reflectogram at the corresponding time delays.

\subsection{Digital Signal Processing for Phase Information Extraction}

Given the reflectogram for the recorded fringes, digital signal processing is performed in order to extract the amplitude and phase response of the DUT whose signature is localized at the position $\tau_{0}$. First of all, the negative frequencies part of the reflectogram is suppressed by setting the corresponding data points to zero (or equivalently after an Hilbert transform). Additionally, a digital band pass filter centered at $\tau_{0}$ (in time domain) is applied to isolate DUT signature and to reduce as much as possible the white noise (shot-noise plus dark-noise 
contribution) generated by the detector. The filtered data set is finally inverse Fourier transformed. It is worth noting that the employed digital filter width and shape influence, in spectral domain, both the sharpness and the noise level of the retrieved amplitude and phase transfer functions: the larger the filter width, the sharper and noisier the transfer functions will be, and vice-versa.

The amplitude of the DUT transfer function does not present particular difficulties and is obtained by extracting the envelope of a fringe pattern. As an example of amplitude response extraction, Fig. 2 shows the measured transfer function of a $1 \mathrm{THz}$ bandwidth bandpass filter generated with the Waveshaper (blue curve), in comparison with the programmed filter shape (red curve). Conversely, spectral phase response extraction is more delicate and requires additional signal processing since the DUT signal is centered at $\tau_{0}$ (in time domain). As a consequence, a rapidly growing linear phase profile is expected for the computed $\Phi(\nu)$ by virtue of the Shift Theorem of the Fourier transform, which states that a translation in the time domain corresponds to a linear phase factor in the frequency domain. Therefore, subtraction from the unwrapped phase data of the first-order polynomial fit to the data, corresponding to such linear phase factor, is performed in order to suppress additional propagation delay (in DUT surrounding fibers) and, hence, to isolate the phase response of the DUT.

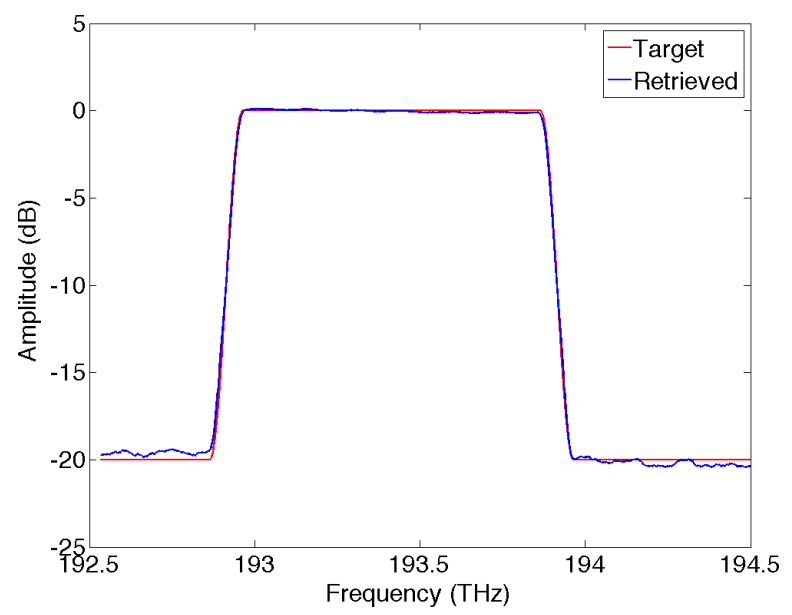

Figure 2. Measured transfer function of a $1 \mathrm{THz}$ bandwidth bandpass filter generated with the Waveshaper (blue), in comparison with the programmed filter shape (red).

Furthermore, for the sake of precision, it is important to remark that not only the phase response of the DUT is encoded in the fringes, but also the group delay dispersion of the optical components that constitute the measurement setup itself. So, in order to precisely determine the phase transfer function of the DUT, the GDD of the setup should be estimated and subtracted from the measured phase profile. For OFDI characterizations in transmission configuration (as in Fig. 1), this can be done by performing a two step measurement: one with the DUT and the other without, and extracting the setup GDD and the overall phase profile using the procedure described above. For measurements in reflection configuration, only one acquisition step is needed; in this case, reflection peaks in the reflectogram corresponding to the input and the output facet of the DUT can be separately used to determine the setup contribution and the overall phase profile (for one round trip in the DUT), respectively. In the present study, characterizations are performed in transmission configuration. However, instead of performing two separate measurements to deduce the phase transfer function of the DUT, we take advantage of the high precision of the programmable optical filter, considering it as an amplitude and phase reference component that we can use to evaluate the phase characterization performances of our OFDR setup with just a single measurement, following the procedure described in sec. 3 .

\subsection{Nonlinear frequency sweep of the tunable laser source}

In deriving the equations governing Coherent OFDI and the signal processing procedure used to extract amplitude and phase information, assumptions have been made concerning the time-linear frequency sweep of the tunable laser source and interferogram sampling at constant frequency spacing. In practice, TLSs frequency sweep may strongly deviate from linearity, so ad hoc techniques are introduced to ensure interferogram sampling at constant 
frequency spacing. Methods typically adopted for this purpose involve the use of an auxiliary interferometer to estimate the time-dependent sweep rate of the laser $\gamma(t)$ and compensate its effect (see Fig. 1). The simplest and most popular way of doing that consists in using the frequency-periodic output of the auxiliary interferometer (cf. eq. 3) as trigger signal for sampling the fringe pattern from the measurement interferometer. ${ }^{16,18}$ This technique allows for fast data acquisition and real-time analysis. However, it is commonly admitted that this method is not necessarily the best choice when measuring phase features. ${ }^{19}$ Alternatively, a different technique consists in sampling with a standard time-domain sampler the output of both interferometers and subsequently performing a numerical resampling at constant frequency spacing of the measurement interferogram, based on the time dependent sweep rate $\gamma(t)$ measured from the auxiliary interferometer fringe pattern. ${ }^{20}$ Compared to the first method, such approach offers a better control of phase information, at the expense of an oversampling of the interferograms and a heavier digital signal processing of the acquired data and it is preferred for our purposes.

\section{SPECTRAL PHASE MEASUREMENT BY OFDR}

We observe from previous sections that a large number of filtering steps are to be applied before data exploitation. Their impact on phase and amplitude characterisation of DUT have not been fully studied in literature. This has motivated the following experimental evaluation of coherent OFDR capabilities when measuring spectral phase/amplitude of DUT. To this end, we investigate the access to the complex transfer function of optical components by using as DUT a commercial optical filter, the Finisar Waveshaper $1000 \mathrm{~S} / \mathrm{X}$, programmable in attenuation and phase over $\mathrm{C}+\mathrm{L}$ band, and following the scheme indicated in Fig. 1. In this section we describe the working principle of such programmable filter and the measurement strategy adopted for our purposes. Finally we present the discrete phase shift and group delay dispersion measurement capabilities of Co-OFDR.

\subsection{Operating Principle of Programmable Filter}

The programmable optical filter Finisar Waveshaper $1000 \mathrm{~S} / \mathrm{X}$, used as reference DUT in the OFDR setup, is a commercial device that can produce user-defined filter shapes with arbitrary amplitude and phase profile and minimum filter bandwidth down to $20 \mathrm{GHz}$. Its operating principle is based on the concept of 4-f Pulse Shaper, first introduced by Froehly, et al. in 1983, ${ }^{21}$ depicted in Fig. 3. It consists in angularly separating the spectral components of the incoming signal by a first grating, and focusing each of them to small diffraction spots in the Fourier plane by a lens. In this plane, a suitably patterned mask can be used to induce optical path length and/or optical density changes for the spatially dispersed spectral components. Shaping of the output waveform is then achieved by recombining the spectral components by a second lens and grating. Such waveform will result from the mapping of the Fourier transform of the mask pattern onto the output spectrum.

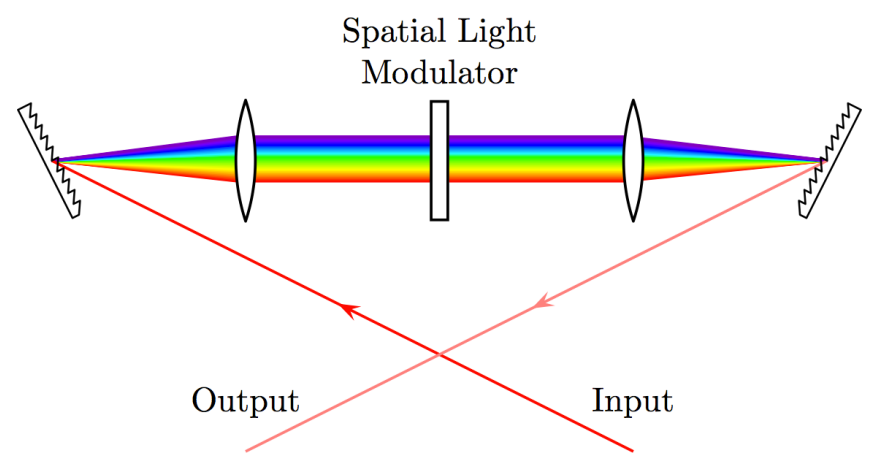

Figure 3. Schematic of the 4-f Pulse Shaper concept.

In the optical filter under consideration, the spatial mask is implemented using a programmable liquid crystal modulator array on silicon substrate (Liquid Crystal on Silicon (LCoS)) providing independent control over spectral amplitude and phase profiles. A polarization diverse optics is used to separate and align the orthogonal polarization states of input field to match the high efficiency $s$-polarization state of the diffraction grating and, 
thus, reduce the polarization-dependent loss of the device. For more details about the implementation and the application in optical fiber communications of this class of LCoS programmable optical processors, the interested reader is referred to Ref. 2 and 22. In the present study, we take advantage of the capabilities of the Waveshaper and, using the digital signal processing procedure described in sec. 2, we investigate the OFDR performances in retrieving the amplitude and phase transfer function programmed on the optical filter. In particular, we focus our attention on the characterization of discrete phase shifts and group delay dispersion.

\subsection{Discrete Phase Shift Measurement}

The capability of OFDR of measuring discrete phase shifts is here evaluated using all-pass optical filters with phase shifts of different amounts applied over $100 \mathrm{GHz}$ bands. The phase response of the filter is characterized using the procedure described in sec. 2: the cumulated delay (or, equivalently, the linear phase factor) due to light propagation in measurement interferometer is systematically compensated. Additionally, the global dispersion introduced by the reference and measurement interferometers is also compensated. To this end, the nearly parabolic phase profile corresponding to the global setup dispersion is estimated by means of polynomial fit to the phase data and subtracted in order to retrieve the correct phase transfer function of the filter. As illustrated in sec. 2, prior to performing such operations, reflectogram data are filtered in time domain, using a narrow digital filter around the peak corresponding to the DUT, in order to reduce as much as possible the white noise contribution in the spectral domain.
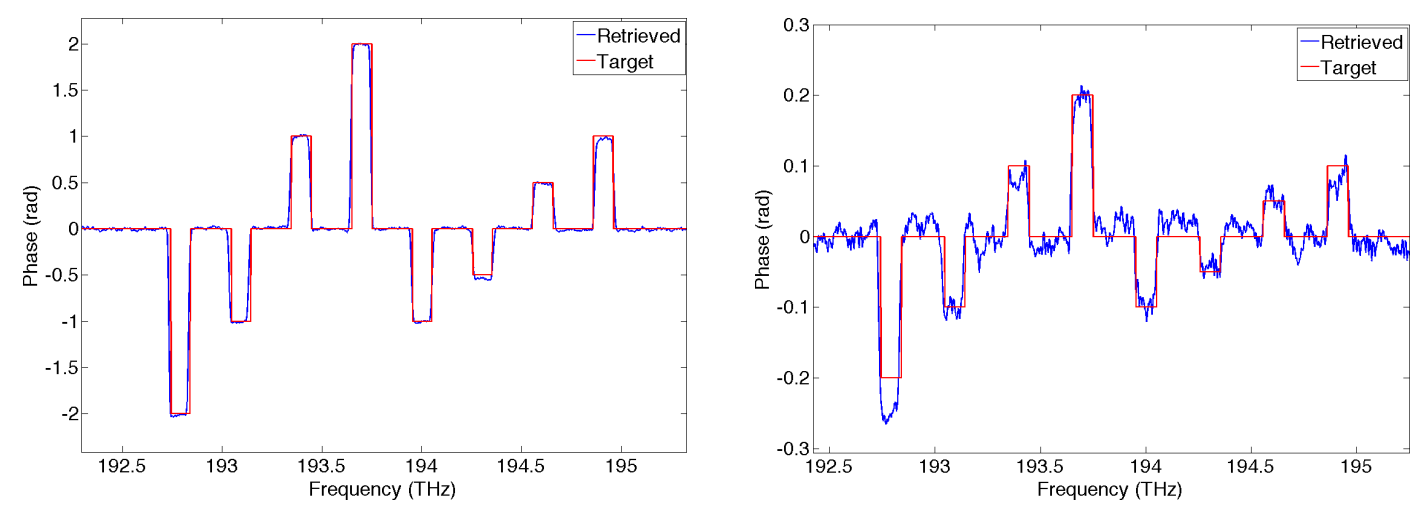

Figure 4. Examples of discrete phase shifts measurements: (a) phase shift values ranging from -2 and 2 rad with minimum step of $0.5 \mathrm{rad}$; (b) phase shift values from -0.2 and $0.2 \mathrm{rad}$ with minimum step of $0.05 \mathrm{rad}$.

Examples of measurements of discrete phase shifts of different amounts are presented in Fig. 4. It is observed that for phase shift of few rads down to several hundreds of mrad, the desired phase profile can be accurately retrieved. However, for phase shifts of several tens of mrad, a noise becomes appearent. Nonetheless, small phase shift down to $50 \mathrm{mrad}$ can be detected by OFDR.

\subsection{Group Delay Dispersion Measurement}

In an analogous way, group delay dispersion measurement capabilities of OFDR are evaluated by imposing a parabolic spectral phase profile to the programmable filter and retrieving it. Such phase profile is programmed on the bandpass of a $1 \mathrm{THz}$ bandwidth filter as that shown in Fig. 2. The dispersion of the OFDR setup is estimated from the phase data set corresponding to the filter stop-band. Examples of dispersion measurements for GDD values of 1, 0.2 and $0.02 \mathrm{ps} / \mathrm{ns}$ are shown in Fig. 5 . As for the case of discrete phase shift measurements, for large values of dispersion the retrieved phase profile accurately reproduces the programmed one. When the dispersion decreases the impact of noise on the measurement becomes more important. Nevertheless, GDD values down to $20 \mathrm{fs} / \mathrm{nm}$ can be measured over an optical bandwidth as narrow as $1 \mathrm{THz}$.

When optical filters with larger bandwidth are considered, it is observed that the noise impacting on dispersion measurements is not a white noise. More precisely, a periodical fluctuation seems to be superimposed to the parabolic phase related to the programmed GDD (see Fig. 5 (right)).

The origin of these fluctuations needs to be be more carefully identified and is studied in the next section. 

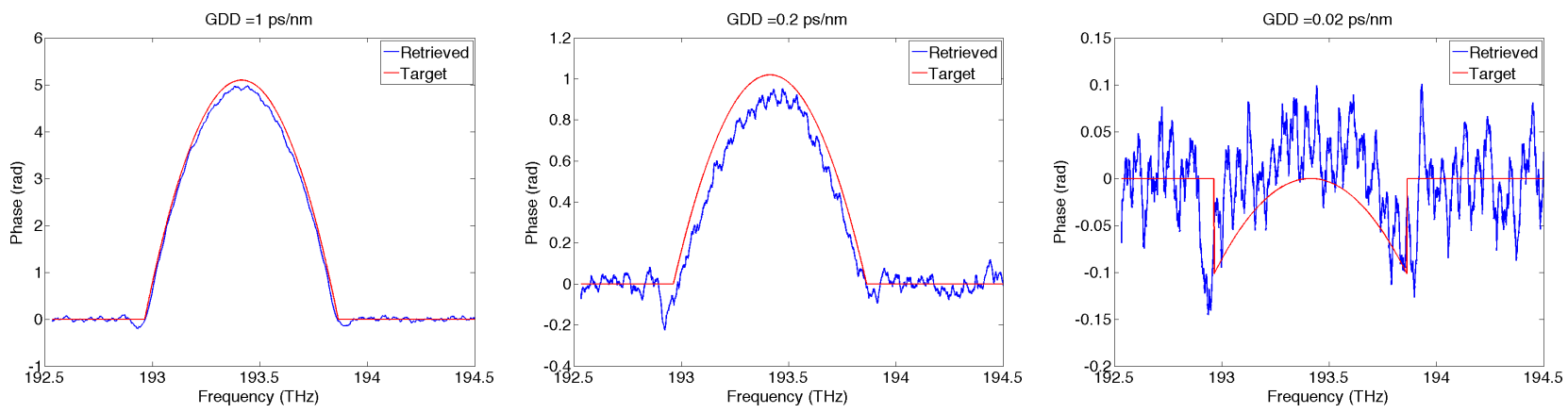

Figure 5. Examples of group delay dispersion measurements for GDD values of $1 \mathrm{ps} / \mathrm{nm}$ (left), $0.2 \mathrm{ps} / \mathrm{nm}$ (center) and $0.02 \mathrm{ps} / \mathrm{nm}$ (right).

\section{CONSIDERATIONS ON DOPPLER EFFECT}

\subsection{Specific features of phase fluctuations}

In order to gain more insight into the physical properties of the fluctuation observed in the measurements reported in the previous section, further OFDI characterizations have been performed as a function of the TLS sweep rate. Figure 6 shows the phase profiles and the raw reflectogram data recorded for an all-pass filter shape with a flat phase response, at nominal sweep rates of 10 and $20 \mathrm{~nm} / \mathrm{s}$.
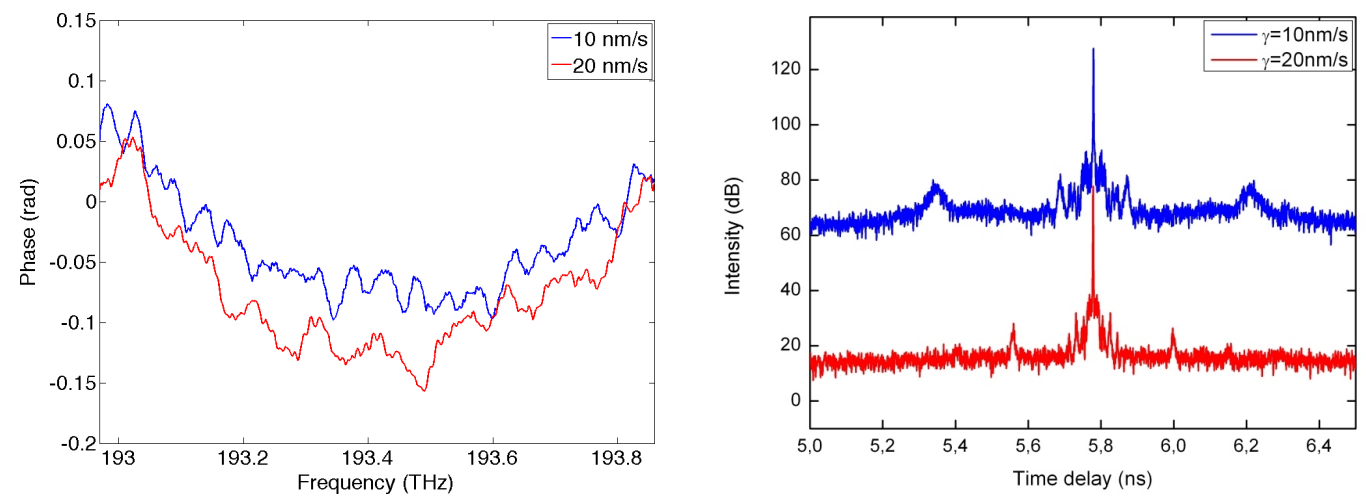

Figure 6. Spectral phase measurements (left) and corresponding reflectograms (right) for nominal sweep rates of 10 and $20 \mathrm{~nm} / \mathrm{s}$.

From fig. 6 two main features can be observed. First of all, the average phase variation over the optical frequency range of the measurement is parabolic. This can be attributed to GDD of the OFDI setup, as explained in section 2. In the second instance, the fluctuations superimposed on the parabolic phase profile (see fig. 6, left) seem to be almost periodical. More importantly, their periods manifest to be highly dependent on the TLS tuning rate. At the same time, two symmetric sidebands are observed on both side of the main peak on the corresponding reflectograms (see fig. 6, right). Their positions are influenced by the tuning rate of the TLS, with a separation relatively to the central peak that decreases when the sweep rate $\gamma$ is increased. These experimental results suggest that the sidebands observed in time-domain as well as the period of the recorded phase fluctuations in spectral domain are closely related one another. Furthermore, since their different characteristics depend on the TLS tuning rate, and consequently, on the time needed to perform an OFDR data acquisition, it becomes clear that the experimental conditions are changing over the data acquisition.

\subsection{Dynamical aspects of OFDR measures}

A phase modulation of light during its transmission through the DUT is a possible hypothesis that can explain the previous observations. To clarify this affirmation, and in complement to usual assumptions that are implicitly imposed when analysing OFDR signals, we consider in the following the situation in which the DUT, or part 
of the OFDR setup, has characteristics that may vary over time. In other words, dynamical aspects occurring during the OFDR data measurement are here specifically addressed. To do so, eq. 2 is reviewed assuming now that the time delay $\tau=\tau_{2}-\tau_{1}$ is not constant anymore but it can change over time. In particular, we consider

$$
\tau(t)=\tau_{0}-\Delta \tau(t)
$$

where $\tau_{0}$ is the time delay of the OFDR measurement interferometer in the absence of perturbations, while $\Delta \tau(t) \ll \tau_{0}$ is a perturbation term that represents the time deviation from the static condition needed for the light to propagate in the OFDR measurement interferometer.

This formulation is quite general and well suited to describe the effect induced by different physical phenomena, such as the refractive variations due to temperature or strain fluctiations in the fibered interferometer, or coupling of mechanical perturbations to the DUT properties. In order to simplify the mathematical study of such situation, a DUT with a flat phase $\Phi(\nu)$ and amplitude $A(\nu)$ response is considered. This choice corresponds to a DUT that introduces neither extra time-delay nor amplitude distortion (in agreement with experimental conditions of measurements reported in fig. 6). With the definition in eq. 4 , interference fringes in equation 2 become,

$$
\tilde{U}(t)=2 U_{0} A \cos \left(4 \pi \gamma\left(\tau_{0}-\Delta \tau(t)\right) t+\varphi\right),
$$

where $\varphi$ is the same phase constant as in equation 2. If we rearrange equation 5 to take into account the impact of variations of $\tau(t)$ with time by further considering $\nu_{0} \gg \gamma t \gg \gamma \tau_{0}$ (since we only address the case of a perturbation), equation 5 becomes:

$$
U(t)=U_{0} \cos \left(4 \pi \gamma \tau_{0} t+\Phi_{m}(t)+\varphi\right),
$$

where

$$
\Phi_{m}(t)=4 \pi \nu_{0} \Delta \tau(t)=4 \pi \frac{\nu_{0}}{c} x(t)
$$

and $x(t)$ represents the optical path change caused by external perturbations.

A close examination of the phase of equation 6 reveals two distinct physical contributions. The first one (i.e. the term that varies linearly with time) corresponds to the relative time delay caused by the propagation of light in the OFDR interferometer under static conditions. The second term $\left(\Phi_{m}(t)\right)$ corresponds to dynamical contribution of the interferometer. It is important to remark that, since the optical path difference of the two arms of the interferometer is not constant by definition, light passing through the interferometer will be affected by Doppler effect and the time-varying instantaneous optical frequency of the TLS will experiences an additional spectral shift. Using eq. 7, the signatures of Doppler effect on OFDI measurement can be conveniently modelled and studied.

\section{a Case of a constant Doppler effect during OFDR data acquisition}

In this section, we consider the most common situation when dealing with Doppler effect, that is when the optical path length linearly changes over the measurement time. In this case, a constant Doppler frequency shift is expected for the light that propagates through the measurement interferometer of the OFDI setup. Such situation is modelled by imposing that $x(t)$ in eq. 7 varies linearly with $t$, i.e. $x(t)=w t$, where $w$ is the rate of change of the optical path length, expressed in $\mathrm{m} / \mathrm{s}$.

Figure 7 shows the simulated reflectograms, obtained for two different values of the velocity $w$ and four different tuning rates $\gamma$ of the TLS, after Fourier transformation of eq. 6 and 7 . In the simulations, the parameter $\tau_{0}$ is set to $5000 \mathrm{~s}$, as can be observed from the curve for $w=0$. It can be noticed that, when Doppler effect is introduced in the simulations (by setting here $w=0.1 \mathrm{~m} / \mathrm{s}$ ), the position of the reflectometer peak is shifted from its nominal position by an amount that strongly depends on the tuning rate parameter $\gamma$. In particular, the higher the tuning rate, the lower the shift of the reflectogram peak. From the analytical expression of the Fourier Transform of eq. 7, the peak shift is calculated to be $\Delta=w / \gamma$.

The simulations reported in fig. 7 show that ambiguities on the optical path length can be encountered when exploiting the OFDR reflectogram data. Indeed, in addition to the ambiguities already identified in ref. 16, we note that a full exploitation of reflectogram abscissa requires some extra knowledge on possible Doppler effects 


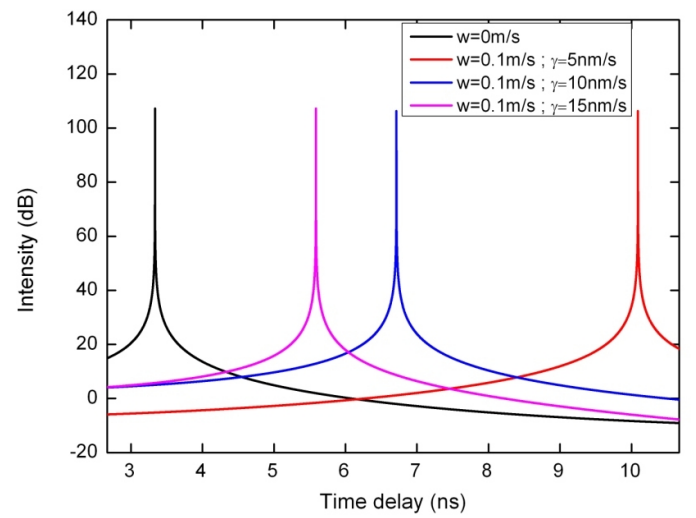

Figure 7. Simulation results of reflectograms in the condition of a constant speed $w=0.1 \mathrm{~m} / \mathrm{s}$ of the reflector for frequency sweep rates of 5,10 , and $15 \mathrm{~nm} / \mathrm{s}$.

present when the measurements are performed. In particular, when the DUT is subject to dynamical phase variation (as is the case, for example, of phase modulators), special care must be taken when exploiting its signature in OFDR reflectogram.

\section{b Case of a non-constant Doppler effect during OFDR data acquisition}

In this section, we examine the most general situation where Doppler effect is not constant during OFDR data acquisition. To this end, let's consider a pure harmonic perturbation described by setting in eq. 7 $x(t)=x_{m} \cos \left(2 \pi \nu_{m} t\right)$, where $x_{m}$ is the amplitude of the phase perturbation and $\nu_{m}$ its frequency. In this case, the temporal evolution of the signal recorded by the detector can be expressed (after some conventional mathematical manipulations of eq. 6) with a series of Bessel functions of the first kind, as:

$$
U(t)=U_{0} \sum_{n=-\infty}^{+\infty} J_{n}\left(\frac{2 \pi x_{m}}{\lambda_{0}}\right) \cos \left[2 \pi\left(\gamma \tau_{0}+n \nu_{m}\right) t+\varphi_{0}\right],
$$

with $\lambda_{0}$ is the wavelength of the TLS. The simulated reflectogram presented in Fig. 8 are obtained after a Fourier transform of eq. 8 for different parameters. When $x_{m}$ is set to 0 (i.e. no Doppler effect is simulated), only one peak is present in the reflectogram, which in this case is identical to that reported in Fig. 7 for $w=0$. On the other hand, when $x_{m} \neq 0$ (i.e. in the case of pure harmonic perturbation), multiple side-peaks, regularly spaced from the main central peak are observed in the simulated OFDR reflectograms. More precisely, the position of the $n$-th peak is separated from the central peak by $n \nu_{m} / \gamma$ and its energy is $J_{n}\left(2 \pi x_{m} / \lambda_{0}\right)$, as indicated by eq. 8 . When OFDI data are represented in the spectral domain, i.e. after an inverse Fourier Transform of reflectogram, the retrieved phase profile appears to be modulated, with a modulation period that strongly depends on the tuning rate of the TLS (see Fig. 8, right).

It is worth to note that, although the previous formulation may appear very specific and restricted to the case of pure harmonic modulation, it is indeed very general and it can be easily extended to the case of non-periodic modulation by virtue of the spectral decomposition by means of the Fourier transform.

We would like to stress that, because of the high sensitivity $S$ and dynamic range $D$ of OFDI measurements (in our case evaluated at $S=-125 \mathrm{~dB}$ and $D=80 \mathrm{~dB}$, respectively) and because of Bessel functions properties, phase perturbation of extremely low amplitude $x_{m}$ (compared to the TLS wavelength) or faint temporal evolution the phase of the DUT can be detected by OFDI. We also further insist on the fact that, without any precise knowledge on the temporal phase characteristics of the DUT, the interpretation of reflectograms peaks, as well as their phase, can be ambiguous. In fact, without any a priori on the nature of the reflectogram peaks, the different side peaks observed in experimental results or simulations can be attributed either to specific reflections in the DUT or to Doppler effect. Nonetheless, the symmetric structure of the modulation sidebands with respect a central, main peak and the tuning rate dependence of the position of the sidebands allow for an easy and fast discrimination of Doppler effect related peaks and reflections in the DUT. This feature enables new applications 

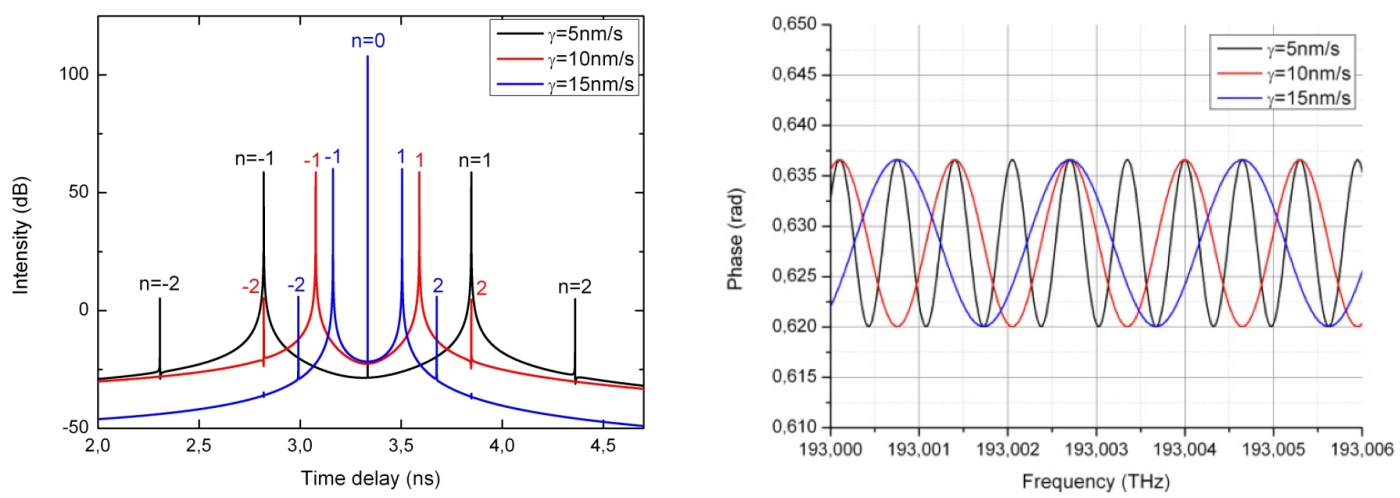

Figure 8. Simulation results showing reflectogram (left) and phase (right) profiles of an output signal phase in the case sinusoidal motion of the reflector of amplitude $x_{m}=1 \mathrm{~nm}$, frequency $\nu_{m}=1 \mathrm{kHz}$, and angle $\alpha=0$.

of OFDR for the characterization of the dynamical properties of devices. In addition, as independent fluctuations acting on different reflectors along the beam path will give rise to different modulation sidebands on both sides of the respective reflection peaks in the reflectogram, OFDR allows the characterization of the temporal phase of multiple target in the direction of the probe beam, overcoming the limitation of techniques such as LDV or Doppler OCT.

We finally note that the simulations carried out are well suited to describe and explain the experimental results reported in fig. 6. In particular, they show that one or more sources of technical noise, inducing phase fluctuations on the OFDI measurement by virtue of the Doppler effect, was present in the environment of the setup or in proximity to the optics of the programmable filter, during the measures. The amplitude of the variation of optical path difference between the two arms of the OFDR measurement interferometer is small, as indicated by the relative amplitude of the modulation sidebands with respect to the main reflectogram peak, and estimated to be on the order of the picometer or below. These measurements further confirm the potential of OFDR technique for the characterization of microscopic optical path length variation and of the dynamical properties of devices.

\section{CONCLUSIONS}

The spectral phase measurement performance of coherent OFDR have been systematically studied by retrieving the phase response of a commercially available optical filter (Finisar Waveshaper 1000S/X) for which the amplitude and phase transfer function can be programmed in the $\mathrm{C}+\mathrm{L}$ band. The technique allows to measure discrete phase shifts down to $50 \mathrm{mrad}$ and group delay dispersions down to $20 \mathrm{fs} / \mathrm{nm}$ over $1 \mathrm{THz}$ optical frequency bandwidth, proving to be a powerful tool for the characterization of amplitude and phase response of optical components. Spectral phase measurement capabilities of OFDR are seen to be limited primarily by phase fluctuations induced by coupling with environmental vibration as a result of Doppler effect. Such phenomenon has a larger impact on OFDR characterizations compared to known measurement ambiguities of the technique (e.g. ghost peaks). However, its occurrence can be easily recognized by the presence of symmetric modulation bands on both sides of a peak in the reflectogram. Such ease of detection enable the use of OFDR for the characterization of both static and dynamical properties of optical components. Moreover, the detection by OFDR of small temporal phase modulations, induced by the microscopic movement of a target in the direction of the probe beam, and the possibility of simultaneously retrieving the instantaneous position of several reflecting target along the beam path pave the way for new application in the fields of distributed sensing and vibration monitoring.

\section{ACKNOWLEDGMENTS}

This work has been supported by the EU FP7 ITN PROPHET and French ANR DIQDOT projects. 


\section{REFERENCES}

1. G. P. Agrawal, J. E. Geusic, and P. J. Anthony, "Distributed feedback lasers with multiple phase-shift regions," Applied Physics Letters 53, pp. 178-179, 1988.

2. M. F. Roelens, S. Frisken, J. Bolger, D. Abakoumov, G. Baxter, S. Poole, and B. Eggleton, "Dispersion trimming in a reconfigurable wavelength selective switch," Journal of Lightwave Technology 26(1), pp. 7378, 2008.

3. T. Herr, K. Hartinger, J. Riemensberger, C. Y. Wang, E. Gavartin, R. Holzwarth, M. L. Gorodetsky, and T. J. Kippenberg, "Universal formation dynamics and noise of Kerr-frequency combs in microresonators," Nature Photonics 6(7), pp. 480-487, 2012.

4. M. Schell, M. Tsuchiya, and T. Kamiya, "Chirp and stability of mode-locked semiconductor lasers," IEEE Journal of Quantum Electronics 32(7), pp. 1180-1190, 1996.

5. S. T. Cundiff and A. M. Weiner, "Optical arbitrary waveform generation," Nature Photonics 4(11), pp. 760766, 2010.

6. R. Trebino, Frequency-Resolved Optical Gating: The Measurement of Ultrashort Laser Pulses, Springer, 2000.

7. C. Dorrer and I. Kang, "Simultaneous temporal characterization of telecommunication optical pulses and modulators by use of spectrograms," Optics Letters 27(15), pp. 1315-1317, 2002.

8. C. Iaconis and I. Walmsley, "Spectral phase interferometry for direct electric-field reconstruction of ultrashort optical pulses," Optics Letters 23(10), pp. 792-794, 1998.

9. D. A. Reid, S. G. Murdoch, and L. P. Barry, "Stepped-heterodyne optical complex spectrum analyzer," Optics Express 18(19), pp. 19724-19731, 2010.

10. S. Diddams and J.-C. Diels, "Dispersion measurements with white-light interferometry," JOSA B 13(6), pp. 1120-1129, 1996.

11. C. Palavicini, Y. Jaouën, G. Debarge, E. Kerrinckx, Y. Quiquempois, M. Douay, C. Lepers, A.-F. Obaton, and G. Melin, "Phase-sensitive optical low-coherence reflectometry technique applied to the characterization of photonic crystal fiber properties," Optics Letters 30(4), pp. 361-363, 2005.

12. K. Naganuma, "Semiconductor laser cavity dispersion measurement based on interferometric crosscorrelation of amplified spontaneous emission," Applied Physics Letters 64(3), pp. 261-263, 1994.

13. C. Palavicini, G. Campuzano, B. Thedrez, Y. Jaouën, and P. Gallion, "Analysis of optical-injected distributed feedback lasers using complex optical low-coherence reflectometry," IEEE Photonics Technology Letters 15(12), pp. 1683-1685, 2003.

14. Y. Gottesman, E. V. K. Rao, H. Sillard, and J. Jacquet, "Modeling of optical low coherence reflectometry recorded bragg reflectograms: Evidence to a decisive role of bragg spectral selectivity," Journal of Lightwave Technology 20(3), pp. 489-493, 2002.

15. A. Parini, P. Hamel, A. De Rossi, S. Combrie, N.-V.-Q. Tran, Y. Gottesman, R. Gabet, A. Talneau, Y. Jaouën, and G. Vadala, "Time-wavelength reflectance maps of photonic crystal waveguides: A new view on disorder-induced scattering," Journal of Lightwave Technology 26(23), pp. 3794-3802, 2008.

16. U. Glombitza and E. Brinkmeyer, "Coherent frequency-domain reflectometry for characterization of singlemode integrated-optical waveguides," Journal of Lightwave Technology 11(8), pp. 1377-1384, 1993.

17. X. Fan, Y. Koshikiya, and F. Ito, "Phase-noise-compensated optical frequency-domain reflectometry," IEEE Journal of Quantum Electronics 45(6), pp. 594-602, 2009.

18. B. J. Soller, D. K. Gifford, M. S. Wolfe, and M. E. Froggatt, "High resolution optical frequency domain reflectometry for characterization of components and assemblies," Optics Express 13(2), pp. 666-674, 2005.

19. T.-J. Ahn, Y. Jung, K. Oh, and D. Y. Kim, "Optical frequency-domain chromatic dispersion measurement method for higher-order modes in an optical fiber," Optics Express 13, pp. 10040-10048, Dec. 2005.

20. T.-J. Ahn, J. Y. Lee, and D. Y. Kim, "Suppression of nonlinear frequency sweep in an optical frequencydomain reflectometer by use of hilbert transformation," Applied Optics 44(35), p. 7630, 2005.

21. C. Froehly, B. Colombeau, and M. Vampouille, "II shaping and analysis of picosecond light pulses," in Progress in Optics, E. Wolf, ed., Volume 20, pp. 63-153, Elsevier, 1983.

22. Finisar, "Waveshaper 1000s programmable optical filter." http://www.finisar.com/products/opticalinstrumentation/WaveShaper-1000S, 2013. 